\title{
A fixed point theorem for generalized $(\psi, \varphi)$-weak contractions in Branciari type generalized metric spaces
}

Zhiqun Xue' and Guiwen LV'*

\section{"Correspondence:}

Ivguiwen@stdu.edu.cn

'Department of Mathematics and

Physics, Shijiazhuang Tiedao

University, Shijiazhuang 050043, P.R. China

\begin{abstract}
In this paper, we obtain a new convergence theorem for fixed points of weak contractions in Branciari type generalized metric spaces under weaker conditions. The proof process of the theorem is new and different from that of other authors. An illustrative example of this theorem is to show how the new conditions extend known results.
\end{abstract}

MSC: Primary $47 \mathrm{H} 10$; secondary $54 \mathrm{H} 25$

Keywords: Generalized metric spaces; Lower limit; Fixed point

\section{Introduction}

The concept of generalized metric spaces, which is a generalization of metric spaces, was first defined by Branciari (see [1]) in 2000. The generalization is via the fact that the triangle inequality is replaced by the rectangular inequality $d(x, y) \leq d(x, u)+d(u, v)+d(v, y)$ for all pairwise distinct points $x, y, u, v$ of $X$. Afterwards, many authors studied and extended the existence of old fixed point results in such spaces (see [1-18]). In this paper, we are to generalize well-known related fixed points theorems. For this, we recall some basic definitions and conclusions.

Definition 1.1 ([1]) Let $X$ be a nonempty set and $d: X \times X \rightarrow[0,+\infty)$ be a mapping such that for all $x, y \in X$ and for all distinct points $u, v \in X$ each of them different from $x$ and $y$ satisfying the following conditions:

$\left(d_{1}\right) d(x, y)=0$ if and only if $x=y$;

$\left(d_{2}\right) d(x, y)=d(y, x)$;

$\left(d_{3}\right) d(x, y) \leq d(x, u)+d(u, v)+d(v, y)$ (the rectangular inequality).

Then $(X, d)$ is called a Branciari type generalized metric space.

Every metric space is a Branciari type generalized metric space, but the converse is not true (see [2]).

(c) The Author(s) 2021. This article is licensed under a Creative Commons Attribution 4.0 International License, which permits use sharing, adaptation, distribution and reproduction in any medium or format, as long as you give appropriate credit to the original author(s) and the source, provide a link to the Creative Commons licence, and indicate if changes were made. The images or other third party material in this article are included in the article's Creative Commons licence, unless indicated otherwise in a credit line to the material. If material is not included in the article's Creative Commons licence and your intended use is not permitted by statutory regulation or exceeds the permitted use, you will need to obtain permission directly from the copyright holder. To view a copy of this licence, visit http://creativecommons.org/licenses/by/4.0/. 
Definition $1.2([1])$ Let $(X, d)$ be a Branciari type generalized metric space and $\left\{x_{n}\right\}$ be a sequence in $X$ and $x \in X$. We call that

(i) $\left\{x_{n}\right\}$ is convergent to $x$ if and only if $d\left(x_{n}, x\right) \rightarrow 0$ as $n \rightarrow \infty$ (denoted by $x_{n} \rightarrow x$ ).

(ii) $\left\{x_{n}\right\}$ is a Cauchy sequence if and only if for each $\epsilon>0$ there exists a natural number $N$ such that $d\left(x_{n}, x_{m}\right)<\epsilon$ for all $m, n>N$.

(iii) $X$ is complete if and only if every Cauchy sequence is convergent in $X$.

In 2012, Lakzian and Samet [4] obtained a fixed point theorem of the generalized metric spaces.

Theorem 1.1 ([4]) Let $(X, d)$ be a Hausdorff and complete generalized metric space, and let $T: X \rightarrow X$ be a self-mapping satisfying

$$
\psi(d(T x, T y)) \leq \psi(d(x, y))-\varphi(d(x, y))
$$

for all $x, y \in X$, where $(i) \psi:[0,+\infty) \rightarrow[0,+\infty)$ is a continuous and monotone nondecreasing function with $\psi(t)=0$ if and only if $t=0 ;(i i) \varphi:[0,+\infty) \rightarrow[0,+\infty)$ is a continuous function with $\varphi(t)=0$ if and only if $t=0$. Then $T$ has a unique fixed point.

In 2013, Liu and Chai [8] gave a generalization of Theorem 1.1.

Theorem 1.2 ([5]) Let $(X, d)$ be a Hausdorff and complete generalized metric space, and let $T: X \rightarrow X$ be a self-mapping satisfying

$$
\begin{aligned}
\psi(d(T x, T y)) \leq & \psi\left(a_{1} d(x, y)+a_{2} d(x, T x)+a_{3} d(y, T y)\right) \\
& -\theta\left(a_{1} d(x, y)+a_{2} d(x, T x)+a_{3} d(y, T y)\right)
\end{aligned}
$$

for all $x, y \in X$, where $(i) \psi:[0,+\infty) \rightarrow[0,+\infty)$ is a continuous and monotone nondecreasing function with $\psi(t)=0$ if and only if $t=0$; (ii) $\theta:[0,+\infty) \rightarrow[0,+\infty)$ satisfies $\lim _{t \rightarrow r} \theta(t)>0$ for $r>0$ and $\lim _{t \rightarrow r} \theta(t)=0$ if and only if $r=0$; (iii) $a_{i} \geq 0(i=1,2,3)$ with $a_{1}+a_{2}+a_{3} \leq 1$. Then $T$ has a unique fixed point.

It is worth mentioning that $\psi:[0,+\infty) \rightarrow[0,+\infty)$ is both continuous and monotone nondecreasing function, but we cannot obtain that $t_{1} \leq t_{2}$ if $\psi\left(t_{1}\right) \leq \psi\left(t_{2}\right)$. In fact, the erroneous conclusion has been widely applied in proofs of the above theorems. This paper is to provide the correct results related to the above theorems and to weaken the conditions of theorems.

\section{The main results}

In this section, we denote by $\Psi$ the set of functions $\psi:[0,+\infty) \rightarrow[0,+\infty)$ satisfying the following conditions:

$\left(a_{1}\right) \psi$ is monotone nondecreasing;

$\left(a_{2}\right) \lim _{t \rightarrow r} \psi(t)>0$ for $r>0$ and $\lim _{t \rightarrow 0^{+}} \psi(t)=0$;

$\left(a_{3}\right) \psi(t)=0$ if and only if $t=0$.

We denote by $\Phi$ the set of functions $\varphi:[0,+\infty) \rightarrow[0,+\infty)$ satisfying the following conditions:

$\left(b_{1}\right) \lim _{t \rightarrow r} \inf \varphi(t)>0$ for each $r>0$; 
$\left(b_{2}\right) \varphi(t) \rightarrow 0$ implies that $t \rightarrow 0$;

$\left(b_{3}\right) \varphi(t)=0$ if and only if $t=0$.

Theorem 2.1 Let $(X, d)$ be a Branciari type complete generalized metric space, and let $T: X \rightarrow X$ be a self-mapping satisfying

$$
\begin{aligned}
\psi(d(T x, T y)) \leq & \psi\left(a_{1} d(x, y)+a_{2} d(x, T x)+a_{3} d(y, T y)\right) \\
& -\varphi\left(a_{1} d(x, y)+a_{2} d(x, T x)+a_{3} d(y, T y)\right)
\end{aligned}
$$

for all $x, y \in X$, where $\psi \in \Psi, \varphi \in \Phi$ and $a_{i} \geq 0(i=1,2,3)$ with $a_{1}+a_{2}+a_{3} \leq 1$. Then $T$ has a unique fixed point.

Proof In order to prove our conclusion, we divide the proof into the following steps.

Step 1. If there exists a fixed point of $T$, the fixed point is unique.

Suppose that there exist two fixed points $p, q$ with $T p=p \neq q=T q$, it means that $d(p, q) \neq 0$. Taking $x=p$ and $y=q$ in (2.1), we have

$$
\begin{aligned}
\psi(d(p, q))= & \psi(d(T p, T q)) \\
\leq & \psi\left(a_{1} d(p, q)+a_{2} d(p, T p)+a_{3} d(q, T q)\right) \\
& -\varphi\left(a_{1} d(p, q)+a_{2} d(p, T p)+a_{3} d(q, T q)\right) \\
= & \psi\left(a_{1} d(p, q)\right)-\varphi\left(a_{1} d(p, q)\right) .
\end{aligned}
$$

If $a_{1}=0$, then $\psi(d(p, q))=0$, i.e., $d(p, q)=0$, which contradicts $d(p, q) \neq 0$. If $a_{1}>0$, we deduce from (2.2) that

$$
\psi(d(p, q))<\psi\left(a_{1} d(p, q)\right) \leq \psi(d(p, q))
$$

is a contradiction and $p=q$. Hence the fixed point is unique.

Step 2. $T$ has the fixed point in $X$.

Let $x_{0} \in X$ and construct a sequence $\left\{x_{n}\right\}$ in $X$ by $x_{n+1}=T x_{n}$ for all $n \geq 0$.

Case 1. $T$ has a periodic point.

Case 1-1. If $x_{n+1}=x_{n}$ for some $n$, then $x_{n}$ is a fixed point of $T$.

For the rest, we assume that $d\left(x_{n+1}, x_{n}\right) \neq 0$ for all $n$.

Case 1-2. If $x_{n+2}=x_{n}$ for some $n$, then $T x_{n}$ is a fixed point of $T$. Suppose it is not true, then $T x_{n} \neq T^{2} x_{n}$, i.e., $d\left(T x_{n}, T^{2} x_{n}\right)>0$, which implies that $\varphi\left(d\left(T x_{n}, T^{2} x_{n}\right)\right)>0$. By (2.1), we have

$$
\begin{aligned}
\psi( & \left.d\left(x_{n}, x_{n+1}\right)\right) \\
= & \psi\left(d\left(T^{2} x_{n}, T x_{n}\right)\right) \\
\leq & \psi\left(a_{1} d\left(x_{n+1}, x_{n}\right)+a_{2} d\left(x_{n+2}, x_{n+1}\right)+a_{3} d\left(x_{n}, x_{n+1}\right)\right) \\
& -\varphi\left(a_{1} d\left(x_{n+1}, x_{n}\right)+a_{2} d\left(x_{n+2}, x_{n+1}\right)+a_{3} d\left(x_{n}, x_{n+1}\right)\right) \\
= & \psi\left(\left(a_{1}+a_{2}+a_{3}\right) d\left(x_{n}, x_{n+1}\right)\right)-\varphi\left(\left(a_{1}+a_{2}+a_{3}\right) d\left(x_{n}, x_{n+1}\right)\right) \\
\leq & \psi\left(d\left(x_{n}, x_{n+1}\right)\right)-\varphi\left(\left(a_{1}+a_{2}+a_{3}\right) d\left(x_{n}, x_{n+1}\right)\right),
\end{aligned}
$$


i.e., $\varphi\left(\left(a_{1}+a_{2}+a_{3}\right) d\left(x_{n}, x_{n+1}\right)\right)=0$. If $\sum_{i=1}^{3} a_{i} \neq 0$, we get that $d\left(x_{n}, x_{n+1}\right)=0$, a contradiction. If $\sum_{i=1}^{3} a_{i}=0$, we get from (2.3) that $\psi\left(d\left(x_{n}, x_{n+1}\right)\right)=0$, i.e., $d\left(x_{n}, x_{n+1}\right)=0$ is a contradiction to the assumption, and so $T x_{n}$ is a fixed point of $T$.

Case 1-3. If there are two natural numbers $m, n$ such that $x_{m}=x_{n}$ with $m-n>2$ and $x_{i} \neq x_{j}$ for all $n \leq i \neq j<m$, we claim that $T^{m-n-1} x_{n}$ is a fixed point of $T$. Suppose that it does not hold, then

$$
T^{m-n-1} x_{n} \neq T^{m-n} x_{n} \quad \Leftrightarrow \quad d\left(T^{m-n-1} x_{n}, T^{m-n} x_{n}\right)>0 \quad \Leftrightarrow \quad d\left(x_{m-1}, x_{m}\right)>0
$$

it implies that

$$
\varphi\left(d\left(T^{m-n-1} x_{n}, T^{m-n} x_{n}\right)\right)>0 .
$$

Again using (2.1), we get

$$
\begin{aligned}
\psi( & \left.d\left(x_{m+1}, x_{m}\right)\right) \\
= & \psi\left(d\left(T x_{m}, T x_{m-1}\right)\right) \\
\leq & \psi\left(a_{1} d\left(x_{m}, x_{m-1}\right)+a_{2} d\left(x_{m+1}, x_{m}\right)+a_{3} d\left(x_{m-1}, x_{m}\right)\right) \\
& -\varphi\left(a_{1} d\left(x_{m}, x_{m-1}\right)+a_{2} d\left(x_{m+1}, x_{m}\right)+a_{3} d\left(x_{m-1}, x_{m}\right)\right) .
\end{aligned}
$$

If $d\left(x_{m}, x_{m-1}\right)<d\left(x_{m+1}, x_{m}\right)$, then

$$
\begin{aligned}
\psi( & \left.d\left(x_{m+1}, x_{m}\right)\right) \\
\leq & \psi\left(\left(a_{1}+a_{2}+a_{3}\right) d\left(x_{m+1}, x_{m}\right)\right) \\
& -\varphi\left(a_{1} d\left(x_{m}, x_{m-1}\right)+a_{2} d\left(x_{m+1}, x_{m}\right)+a_{3} d\left(x_{m-1}, x_{m}\right)\right) \\
\leq & \psi\left(d\left(x_{m+1}, x_{m}\right)\right) \\
& -\varphi\left(a_{1} d\left(x_{m}, x_{m-1}\right)+a_{2} d\left(x_{m+1}, x_{m}\right)+a_{3} d\left(x_{m-1}, x_{m}\right)\right),
\end{aligned}
$$

i.e.,

$$
\varphi\left(a_{1} d\left(x_{m}, x_{m-1}\right)+a_{2} d\left(x_{m+1}, x_{m}\right)+a_{3} d\left(x_{m-1}, x_{m}\right)\right)=0,
$$

that is,

$$
\left(a_{1}+a_{3}\right) d\left(x_{m}, x_{m-1}\right)+a_{2} d\left(x_{m+1}, x_{m}\right)=0,
$$

which implies that $a_{1}=a_{2}=a_{3}=0$. From (2.4), we obtain that $\psi\left(d\left(x_{m+1}, x_{m}\right)\right)=0 \Leftrightarrow$ $d\left(x_{m+1}, x_{m}\right)=0$, which is a contradiction and so $d\left(x_{m+1}, x_{m}\right) \leq d\left(x_{m}, x_{m-1}\right)$. Then we have

$$
\begin{aligned}
\psi & \left(d\left(x_{n+1}, x_{n}\right)\right) \\
& =\psi\left(d\left(x_{m+1}, x_{m}\right)\right) \\
& =\psi\left(d\left(T x_{m}, T x_{m-1}\right)\right) \\
& \leq \psi\left(a_{1} d\left(x_{m}, x_{m-1}\right)+a_{2} d\left(x_{m}, x_{m+1}\right)+a_{3} d\left(x_{m-1}, x_{m}\right)\right)
\end{aligned}
$$




$$
\begin{aligned}
& -\varphi\left(a_{1} d\left(x_{m}, x_{m-1}\right)+a_{2} d\left(x_{m}, x_{m+1}\right)+a_{3} d\left(x_{m-1}, x_{m}\right)\right) \\
\leq & \psi\left(\left(a_{1}+a_{2}+a_{3}\right) d\left(x_{m-1}, x_{m}\right)\right) \\
& -\varphi\left(a_{1} d\left(x_{m}, x_{m-1}\right)+a_{2} d\left(x_{m}, x_{m+1}\right)+a_{3} d\left(x_{m-1}, x_{m}\right)\right) \\
\leq & \psi\left(d\left(x_{m}, x_{m-1}\right)\right) \\
& -\varphi\left(a_{1} d\left(x_{m}, x_{m-1}\right)+a_{2} d\left(x_{m}, x_{m+1}\right)+a_{3} d\left(x_{m-1}, x_{m}\right)\right),
\end{aligned}
$$

then $a_{1} d\left(x_{m}, x_{m-1}\right)+a_{2} d\left(x_{m}, x_{m+1}\right)+a_{3} d\left(x_{m-1}, x_{m}\right)>0$. Otherwise, $a_{1}=a_{2}=a_{3}=0$, we obtain a contradiction. Therefore, (2.6) turns into

$$
\begin{aligned}
\psi( & \left.d\left(x_{n+1}, x_{n}\right)\right) \\
\leq & \psi\left(d\left(x_{m}, x_{m-1}\right)\right) \\
& \quad-\varphi\left(a_{1} d\left(x_{m}, x_{m-1}\right)+a_{2} d\left(x_{m}, x_{m+1}\right)+a_{3} d\left(x_{m-1}, x_{m}\right)\right) \\
& <\psi\left(d\left(x_{m}, x_{m-1}\right)\right) \\
& \quad . \\
\leq & \psi\left(d\left(x_{n+1}, x_{n}\right)\right)
\end{aligned}
$$

a contradiction. Hence, the conclusion holds.

Case 2. $T$ has no periodic point, i.e., $x_{m} \neq x_{n}$ for all $m \neq n$.

Step 2-1. Show that $\lim _{n \rightarrow \infty} d\left(x_{n+1}, x_{n}\right)=0$. Taking $x=x_{n}, y=x_{n-1}$ in $(2.1)$, we have

$$
\begin{aligned}
\psi( & \left.d\left(x_{n+1}, x_{n}\right)\right) \\
= & \psi\left(d\left(T x_{n}, T x_{n-1}\right)\right) \\
\leq & \psi\left(a_{1} d\left(x_{n}, x_{n-1}\right)+a_{2} d\left(x_{n}, x_{n+1}\right)+a_{3} d\left(x_{n-1}, x_{n}\right)\right) \\
& -\varphi\left(a_{1} d\left(x_{n}, x_{n-1}\right)+a_{2} d\left(x_{n}, x_{n+1}\right)+a_{3} d\left(x_{n-1}, x_{n}\right)\right) .
\end{aligned}
$$

If $d\left(x_{n}, x_{n-1}\right)<d\left(x_{n+1}, x_{n}\right)$, then

$$
\begin{aligned}
& \psi\left(d\left(x_{n+1}, x_{n}\right)\right) \\
& \quad \leq \psi\left(d\left(x_{n+1}, x_{n}\right)\right) \\
& \quad-\varphi\left(a_{1} d\left(x_{n}, x_{n-1}\right)+a_{2} d\left(x_{n}, x_{n+1}\right)+a_{3} d\left(x_{n-1}, x_{n}\right)\right),
\end{aligned}
$$

it implies that

$$
\varphi\left(a_{1} d\left(x_{n}, x_{n-1}\right)+a_{2} d\left(x_{n}, x_{n+1}\right)+a_{3} d\left(x_{n-1}, x_{n}\right)\right)=0
$$

then $a_{1}=a_{2}=a_{3}$. Thus

$$
\psi\left(d\left(x_{n+1}, x_{n}\right)\right)=0 \quad \Leftrightarrow \quad d\left(x_{n+1}, x_{n}\right)=0,
$$

a contradiction. Hence

$$
d\left(x_{n+1}, x_{n}\right) \leq d\left(x_{n}, x_{n-1}\right)
$$


for all $n$. Since $\psi$ is monotone nondecreasing, then

$$
\psi\left(d\left(x_{n+1}, x_{n}\right)\right) \leq \psi\left(d\left(x_{n}, x_{n-1}\right)\right) .
$$

There exist nonnegative numbers $r$ and $r^{*}$ such that

$$
\lim _{n \rightarrow \infty} d\left(x_{n+1}, x_{n}\right)=r, \quad \lim _{n \rightarrow \infty} \psi\left(d\left(x_{n+1}, x_{n}\right)\right)=r^{*} .
$$

If $r>0$, we get

$$
\begin{aligned}
& \lim _{n \rightarrow \infty}\left[a_{1} d\left(x_{n}, x_{n-1}\right)+a_{2} d\left(x_{n}, x_{n+1}\right)+a_{3} d\left(x_{n-1}, x_{n}\right)\right] \\
& \quad=\left(a_{1}+a_{2}+a_{3}\right) r>0,
\end{aligned}
$$

then

$$
\lim _{n \rightarrow \infty} \inf \varphi\left(a_{1} d\left(x_{n}, x_{n-1}\right)+a_{2} d\left(x_{n}, x_{n+1}\right)+a_{3} d\left(x_{n-1}, x_{n}\right)\right)>0 .
$$

By (2.8), we have

$$
\begin{aligned}
\psi & \left(d\left(x_{n+1}, x_{n}\right)\right) \\
\leq & \psi\left(d\left(x_{n}, x_{n-1}\right)\right) \\
& -\varphi\left(a_{1} d\left(x_{n}, x_{n-1}\right)+a_{2} d\left(x_{n}, x_{n+1}\right)+a_{3} d\left(x_{n-1}, x_{n}\right)\right) .
\end{aligned}
$$

Letting $n \rightarrow \infty$ in (2.13), taking lower limits on each side of the above inequality, we deduce that

$$
\lim _{n \rightarrow \infty} \inf \varphi\left(a_{1} d\left(x_{n}, x_{n-1}\right)+a_{2} d\left(x_{n}, x_{n+1}\right)+a_{3} d\left(x_{n-1}, x_{n}\right)\right) \leq 0,
$$

a contradiction, and so $\lim _{n \rightarrow \infty} d\left(x_{n+1}, x_{n}\right)=0$.

Step 2-2. Show that $\lim _{n \rightarrow \infty} d\left(x_{n+2}, x_{n}\right)=0$. Again taking $x=x_{n+1}, y=x_{n-1}$ in (2.1), then

$$
\begin{aligned}
\psi( & \left.d\left(x_{n+2}, x_{n}\right)\right) \\
= & \psi\left(d\left(T x_{n+1}, T x_{n-1}\right)\right) \\
\leq & \psi\left(a_{1} d\left(x_{n+1}, x_{n-1}\right)+a_{2} d\left(x_{n+2}, x_{n+1}\right)+a_{3} d\left(x_{n-1}, x_{n}\right)\right) \\
& -\varphi\left(a_{1} d\left(x_{n+1}, x_{n-1}\right)+a_{2} d\left(x_{n+2}, x_{n+1}\right)+a_{3} d\left(x_{n-1}, x_{n}\right)\right) \\
\leq & \psi\left(a_{1} d\left(x_{n+1}, x_{n-1}\right)+a_{2} d\left(x_{n-1}, x_{n}\right)+a_{3} d\left(x_{n-1}, x_{n}\right)\right) \\
& -\varphi\left(a_{1} d\left(x_{n+1}, x_{n-1}\right)+a_{2} d\left(x_{n+2}, x_{n+1}\right)+a_{3} d\left(x_{n-1}, x_{n}\right)\right) .
\end{aligned}
$$

If $\sum_{i=1}^{3} a_{i}=0$, then $a_{i}=0$ for $i=1,2,3$. Thus, $\psi\left(d\left(x_{n+2}, x_{n}\right)\right)=0$, a contradiction. If $\sum_{i=1}^{3} a_{i} \neq$ 0 , we consider the following cases. 
Case 2-2-1. If there exists a infinite subsequence $\left\{x_{n(i)}\right\}$ of $\left\{x_{n}\right\}$ such that $d\left(x_{n(i)}, x_{n(i)-1}\right)<$ $d\left(x_{n(i)+1}, x_{n(i)-1}\right)$ for all $i$. Without loss of generality, we have

$$
\begin{aligned}
d\left(x_{n(i)}, x_{n(i)-2}\right) & \leq d\left(x_{n(i)-1}, x_{n(i)-2}\right) \\
& \leq d\left(x_{n(i)-2}, x_{n(i)-3}\right) \\
& \leq \cdots \cdots \\
& \leq d\left(x_{n(i-1)}, x_{n(i-1)-1}\right) \\
& <d\left(x_{n(i-1)+1}, x_{n(i-1)-1}\right)
\end{aligned}
$$

for all $i \geq 1$. Again by (2.1), we get

$$
\begin{aligned}
& \psi\left(d\left(x_{n(i)+1}, x_{n(i)-1}\right)\right) \\
&=\psi\left(d\left(T x_{n(i)}, T x_{n(i)-2}\right)\right) \\
& \leq \psi\left(a_{1} d\left(x_{n(i)}, x_{n(i)-2}\right)+a_{2} d\left(x_{n(i)+1}, x_{n(i)}\right)\right. \\
&\left.+a_{3} d\left(x_{n(i)-1}, x_{n(i)-2}\right)\right)-\varphi\left(a_{1} d\left(x_{n(i)}, x_{n(i)-2}\right)\right. \\
&\left.+a_{2} d\left(x_{n(i)+1}, x_{n(i)}\right)+a_{3} d\left(x_{n(i)-1}, x_{n(i)-2}\right)\right) \\
& \leq \psi\left(\left(a_{1}+a_{2}+a_{3}\right) d\left(x_{n(i)-1}, x_{n(i)-2}\right)\right) \\
&-\varphi\left(a_{1} d\left(x_{n(i)}, x_{n(i)-2}\right)+a_{2} d\left(x_{n(i)+1}, x_{n(i)}\right)\right. \\
&\left.+a_{3} d\left(x_{n(i)-1}, x_{n(i)-2}\right)\right) \\
& \leq \psi\left(d\left(x_{n(i)-1}, x_{n(i)-2}\right)\right)-\varphi\left(a_{1} d\left(x_{n(i)}, x_{n(i)-2}\right)\right. \\
&\left.+a_{2} d\left(x_{n(i)+1}, x_{n(i)}\right)+a_{3} d\left(x_{n(i)-1}, x_{n(i)-2}\right)\right)
\end{aligned}
$$

for all $i$. If $\sum_{i=1}^{3} a_{i}=0$, then $a_{i}=0$ for $i=1,2,3$. Thus, we have $\psi\left(d\left(x_{n(i)+1}, x_{n(i)-1}\right)\right)=0$, i.e., $d\left(x_{n(i)+1}, x_{n(i)-1}\right)=0$ is a contradiction. If $\sum_{i=1}^{3} a_{i} \neq 0$, then we get from (2.16) and (2.15) that

$$
\begin{aligned}
\psi( & \left(d\left(x_{n(i)+1}, x_{n(i)-1}\right)\right) \\
\leq & \psi\left(d\left(x_{n(i)-1}, x_{n(i)-2}\right)\right)-\varphi\left(a_{1} d\left(x_{n(i)}, x_{n(i)-2}\right)\right. \\
& \left.+a_{2} d\left(x_{n(i)+1}, x_{n(i)}\right)+a_{3} d\left(x_{n(i)-1}, x_{n(i)-2}\right)\right) \\
& <\psi\left(d\left(x_{n(i)-1}, x_{n(i)-2}\right)\right) \\
& \ldots \\
\leq & \psi \\
\leq & \psi \\
\leq & \left(d\left(x_{n(i-1)}, x_{n(i-1)-1}\right)\right)
\end{aligned}
$$

It follows from (2.17) and the result of Step 2-1 that

$$
\psi\left(d\left(x_{n(i)+1}, x_{n(i)-1}\right)\right)<\psi\left(d\left(x_{n(i)-1}, x_{n(i)-2}\right)\right) \rightarrow 0
$$


as $i \rightarrow \infty$, that is,

$$
\lim _{i \rightarrow \infty} \psi\left(d\left(x_{n(i)+1}, x_{n(i)-1}\right)\right)=0 .
$$

And we also obtain from (2.17) that

$$
\psi\left(d\left(x_{n(i)+1}, x_{n(i)-1}\right)\right)<\psi\left(d\left(x_{n(i-1)+1}, x_{n(i-1)-1}\right)\right),
$$

which implies that

$$
d\left(x_{n(i)+1}, x_{n(i)-1}\right)<d\left(x_{n(i-1)+1}, x_{n(i-1)-1}\right),
$$

so the sequence $\left\{d\left(x_{n(i)+1}, x_{n(i)-1}\right)\right\}$ is monotone decreasing and bounded below, there exists $R \geq 0$ such that

$$
\lim _{i \rightarrow \infty} d\left(x_{n(i)+1}, x_{n(i)-1}\right)=R
$$

If $R>0$, then

$$
\lim _{i \rightarrow \infty} \psi\left(d\left(x_{n(i)+1}, x_{n(i)-1}\right)\right)>0
$$

contradicts (2.18). Thus $d\left(x_{n(i)+1}, x_{n(i)-1}\right) \rightarrow 0$ as $i \rightarrow \infty$.

Case 2-2-2. If there exists an infinite subsequence $\left\{x_{n(j)}\right\}$ of $\left\{x_{n}\right\}$ such that

$$
d\left(x_{n(j)+1}, x_{n(j)-1}\right) \leq d\left(x_{n(j)}, x_{n(j)-1}\right)
$$

then $d\left(x_{n(j)+1}, x_{n(j)-1}\right) \rightarrow 0$ as $j \rightarrow \infty$.

Therefore, in two cases we proved that $\lim _{n \rightarrow \infty} d\left(x_{n+2}, x_{n}\right)=0$.

Step 2-3. Show that $\left\{x_{n}\right\}$ is a Cauchy sequence. Suppose, on the contrary, that there exists $\epsilon>0$ for which we can find subsequences $\left\{x_{m(k)}\right\}$ and $\left\{x_{n(k)}\right\}$ of $\left\{x_{n}\right\}$ such that

$$
d\left(x_{m(k)}, x_{n(k)}\right) \geq \epsilon
$$

for $n(k)>m(k)>k$ with $n(k)$ is the smallest index, and so we have

$$
d\left(x_{m(k)}, x_{n(k)-1}\right)<\epsilon
$$

for all $k$. Applying the rectangular inequality, we obtain that

$$
\begin{aligned}
\epsilon & \leq d\left(x_{m(k)}, x_{n(k)}\right) \\
& \leq d\left(x_{m(k)}, x_{n(k)-1}\right)+d\left(x_{n(k)-1}, x_{n(k)-2}\right)+d\left(x_{n(k)-2}, x_{n(k)}\right) \\
& <\epsilon+d\left(x_{n(k)-1}, x_{n(k)-2}\right)+d\left(x_{n(k)-2}, x_{n(k)}\right),
\end{aligned}
$$

then $d\left(x_{m(k)}, x_{n(k)}\right) \rightarrow \epsilon$ as $k \rightarrow \infty$. Similarly,

$$
d\left(x_{m(k)}, x_{n(k)}\right)-d\left(x_{m(k)}, x_{m(k)-1}\right)-d\left(x_{n(k)-1}, x_{n(k)}\right)
$$




$$
\begin{aligned}
& \leq d\left(x_{m(k)-1}, x_{n(k)-1}\right) \\
& \leq d\left(x_{m(k)-1}, x_{m(k)}\right)+d\left(x_{m(k)}, x_{n(k)}\right)+d\left(x_{n(k)}, x_{n(k)-1}\right)
\end{aligned}
$$

then $d\left(x_{m(k)-1}, x_{n(k)-1}\right) \rightarrow \epsilon$ as $k \rightarrow \infty$. Furthermore, there exists $K$ such that

$$
d\left(x_{m(k)-1}, x_{n(k)-1}\right)>\frac{\epsilon}{2}, \quad d\left(x_{m(k)}, x_{m(k)-1}\right)<\frac{\epsilon}{2}, \quad d\left(x_{n(k)}, x_{n(k)-1}\right)<\frac{\epsilon}{2}
$$

for $m(k), n(k)>K$. Again using (2.1), then

$$
\begin{aligned}
& \psi\left(d\left(x_{m(k)}, x_{n(k)}\right)\right) \\
&=\psi\left(d\left(T x_{m(k)-1}, T x_{n(k)-1}\right)\right) \\
& \leq \psi\left(a_{1} d\left(x_{m(k)-1}, x_{n(k)-1}\right)+a_{2} d\left(x_{m(k)}, x_{m(k)-1}\right)\right. \\
&\left.\quad+a_{3} d\left(x_{n(k)}, x_{n(k)-1}\right)\right)-\varphi\left(a_{1} d\left(x_{m(k)-1}, x_{n(k)-1}\right)\right. \\
&\left.\quad+a_{2} d\left(x_{m(k)}, x_{m(k)-1}\right)+a_{3} d\left(x_{n(k)}, x_{n(k)-1}\right)\right) \\
& \leq \psi\left(\left(a_{1}+a_{2}+a_{3}\right) d\left(x_{m(k)-1}, x_{n(k)-1}\right)\right) \\
&-\varphi\left(a _ { 1 } d \left(x_{m(k)-1}, x_{n(k)-1}+a_{2} d\left(x_{m(k)}, x_{m(k)-1}\right)\right.\right. \\
&\left.+a_{3} d\left(x_{n(k)}, x_{n(k)-1}\right)\right) \\
& \leq \psi\left(d\left(x_{m(k)-1}, x_{n(k)-1}\right)\right)-\varphi\left(a_{1} d\left(x_{m(k)-1}, x_{n(k)-1}\right)\right. \\
&\left.+a_{2} d\left(x_{m(k)}, x_{m(k)-1}\right)+a_{3} d\left(x_{n(k)}, x_{n(k)-1}\right)\right) .
\end{aligned}
$$

Taking the lower limit as $n \rightarrow \infty$ in the above inequality, (2.19) yields

$$
\begin{aligned}
& \lim _{k \rightarrow \infty} \inf \varphi\left(a_{1} d\left(x_{m(k)-1}, x_{n(k)-1}\right)+a_{2} d\left(x_{m(k)}, x_{m(k)-1}\right)\right. \\
& \left.\quad+a_{3} d\left(x_{n(k)}, x_{n(k)-1}\right)\right) \leq 0 .
\end{aligned}
$$

On the other hand,

$$
\begin{aligned}
& \lim _{k \rightarrow \infty}\left[a_{1} d\left(x_{m(k)-1}, x_{n(k)-1}\right)+a_{2} d\left(x_{m(k)}, x_{m(k)-1}\right)\right. \\
& \left.+a_{3} d\left(x_{n(k)}, x_{n(k)-1}\right)\right]=a_{1} \epsilon .
\end{aligned}
$$

If $a_{1}=0$, then we obtain from (2.19) that

$$
\begin{aligned}
\psi( & \left.d\left(x_{m(k)}, x_{n(k)}\right)\right) \\
\leq & \psi\left(a_{1} d\left(x_{m(k)-1}, x_{n(k)-1}\right)+a_{2} d\left(x_{m(k)}, x_{m(k)-1}\right)\right. \\
& \left.\quad+a_{3} d\left(x_{n(k)}, x_{n(k)-1}\right)\right) \\
& \rightarrow 0
\end{aligned}
$$


as $k \rightarrow \infty$, i.e., $\lim _{k \rightarrow \infty} \psi\left(d\left(x_{m(k)}, x_{n(k)}\right)\right)=0$, a contradiction. If $a_{1} \neq 0$, then (2.20) implies that

$$
\begin{aligned}
& \lim _{k \rightarrow \infty} \inf \varphi\left(a_{1} d\left(x_{m(k)-1}, x_{n(k)-1}\right)+a_{2} d\left(x_{m(k)}, x_{m(k)-1}\right)\right. \\
& \left.\quad+a_{3} d\left(x_{n(k)}, x_{n(k)-1}\right)\right)>0
\end{aligned}
$$

which is a contradiction. Therefore, $\left\{x_{n}\right\}$ is a Cauchy sequence. Since $(X, d)$ is complete, there exists $q \in X$ such that $\lim _{n \rightarrow \infty} x_{n}=q$.

Step 2-4. Let us show that $q$ is a fixed point of $T$. Suppose that it is not the case, then $q$ is not a fixed point of $T$, i.e., $d(q, T q)>0$. Since

$$
\begin{aligned}
d(q, T q)-d\left(q, x_{n}\right)-d\left(x_{n}, x_{n+1}\right) & \leq d\left(T q, T x_{n}\right) \\
& \leq d(T q, q)+d\left(q, x_{n}\right)+d\left(x_{n}, x_{n+1}\right)
\end{aligned}
$$

then

$$
\lim _{n \rightarrow \infty} d\left(T q, x_{n+1}\right)=d(T q, q)>0
$$

Thus,

$$
\lim _{n \rightarrow \infty} \psi\left(d\left(T q, x_{n+1}\right)\right)>0
$$

By (2.1), we get

$$
\begin{aligned}
\psi( & \left.d\left(T q, x_{n+1}\right)\right) \\
= & \psi\left(d\left(T q, T x_{n}\right)\right) \\
\leq & \psi\left(a_{1} d\left(q, x_{n}\right)+a_{2} d(q, T q)+a_{3} d\left(x_{n+1}, x_{n}\right)\right) \\
& -\varphi\left(a_{1} d\left(q, x_{n}\right)+a_{2} d(q, T q)+a_{3} d\left(x_{n+1}, x_{n}\right)\right) .
\end{aligned}
$$

If $a_{2}=0$, then (2.23) yields

$$
\psi\left(d\left(T q, x_{n+1}\right)\right) \leq \psi\left(a_{1} d\left(q, x_{n}\right)+a_{3} d\left(x_{n+1}, x_{n}\right)\right) \rightarrow 0
$$

as $n \rightarrow \infty$, i.e., $\lim _{n \rightarrow \infty} \psi\left(d\left(T q, x_{n+1}\right)\right)=0$, a contradiction. If $a_{2} \neq 0$, then we have

$$
\lim _{n \rightarrow \infty} \inf \varphi\left(a_{1} d\left(q, x_{n}\right)+a_{2} d(q, T q)+a_{3} d\left(x_{n+1}, x_{n}\right)\right)>0
$$

And we get from (2.23) that

$$
\begin{aligned}
& \psi\left(d\left(T q, x_{n+1}\right)\right) \\
& \leq \psi\left(a_{1} d\left(q, x_{n}\right)+d(q, T q)+a_{3} d\left(x_{n+1}, x_{n}\right)\right) \\
&-\varphi\left(a_{1} d\left(q, x_{n}\right)+a_{2} d(q, T q)+a_{3} d\left(x_{n+1}, x_{n}\right)\right) .
\end{aligned}
$$


Since

$$
\lim _{n \rightarrow \infty}\left[a_{1} d\left(q, x_{n}\right)+d(q, T q)+a_{3} d\left(x_{n+1}, x_{n}\right)\right]=d(T q, q)
$$

then

$$
\lim _{n \rightarrow \infty} \psi\left(a_{1} d\left(q, x_{n}\right)+d(q, T q)+a_{3} d\left(x_{n+1}, x_{n}\right)\right)=\lim _{n \rightarrow \infty} \psi\left(d\left(T q, x_{n+1}\right)\right) .
$$

Taking lower limits as $n \rightarrow \infty$ on either side of inequality (2.25), then

$$
\lim _{n \rightarrow \infty} \inf \varphi\left(a_{1} d\left(q, x_{n}\right)+a_{2} d(q, T q)+a_{3} d\left(x_{n+1}, x_{n}\right)\right)=0
$$

which is a contradiction, and hence $q=T q$.

Remark 2.1 If $a_{1}=1, a_{2}=a_{3}=0$ and $\psi, \varphi$ are all continuous in Theorem 2.1, then we obtain Theorem 2.1 of [4]. If $\psi$ is continuous and $\varphi$ has limit in Theorem 2.1, then we obtain the main results of [8].

Remark 2.2 In the proofs of the main theorems of [4, 19], and [8], there exists a common problem for monotonicity of function $\psi$, which is unreasonable that $\psi\left(t_{1}\right) \leq \psi\left(t_{2}\right)$ implies that $t_{1} \leq t_{2}$. It is as follows:

(i) In page 3 of [19], (2.2) implies (2.3).

(ii) In line 5 to line 3 from the bottom of page 903 of [4] and in line 7 to 10 from the top of page 904 of [4].

(iii) The above problems still exist in the proof of the main theorem in [8].

In addition, the proof process of Theorem 2.1 is different from that of $[4,19]$, and [8].

As a corollary of Theorem 2.1, taking $a_{1}=1, a_{2}=a_{3}=0$, we obtain the following result.

Corollary 2.1 Let $(X, d)$ be a Branciari type complete generalized metric space, and let $T: X \rightarrow X$ be a self-mapping satisfying

$$
\psi(d(T x, T y)) \leq \psi(d(x, y))-\varphi(d(x, y))
$$

for all $x, y \in X$, where $\psi$ and $\varphi$ are defined as in Theorem 2.1. Then $T$ has a unique fixed point.

Similar results are obtained from Theorem 2.1 putting $a_{1}=a_{3}=0, a_{2}=1$ or $a_{1}=a_{2}=0$, $a_{3}=1$.

Corollary 2.2 Let $(X, d)$ be a Branciari type complete generalized metric space, and let $T: X \rightarrow X$ be a self-mapping satisfying

$$
\begin{aligned}
& \psi(d(T x, T y)) \leq \psi(d(x, T x))-\varphi(d(x, T x)) \\
& \text { or } \\
& \psi(d(T x, T y)) \leq \psi(d(y, T y))-\varphi(d(y, T y))
\end{aligned}
$$


for all $x, y \in X$, where $\psi$ and $\varphi$ are defined as in Theorem 2.1. Then $T$ has a unique fixed point.

Further, we obtain the following result which includes Corollary 5 of [7].

Corollary 2.3 Let $(X, d)$ be a Branciari type complete generalized metric space, and let $T: X \rightarrow X$ be a self-mapping satisfying

$$
\begin{aligned}
\psi(d(T x, T y)) \leq & \psi(\max \{d(x, y), d(x, T x), d(y, T y)\}) \\
& -\varphi(\max \{d(x, y), d(x, T x), d(y, T y)\})
\end{aligned}
$$

for all $x, y \in X$, where $\psi$ and $\varphi$ are defined as in Theorem 2.1. Then $T$ has a unique fixed point.

Finally, we introduce a simple example [7] that supports the result of our Theorem 2.1.

Example 2.1 ([7]) Let $X=A \cup B$, where $A=\left\{\frac{1}{2}, \frac{1}{3}, \frac{1}{4}, \frac{1}{5}\right\}$ and $B=[1,2]$. Define the generalized metric on $X$ as follows:

$$
d(x, y)= \begin{cases}d(y, x), & x, y \in X, \\ 0, & x, y \in X \text { with } x=y, \\ 0.3, & x=\frac{1}{2}, y=\frac{1}{3} \text { or } x=\frac{1}{4}, y=\frac{1}{5}, \\ 0.2, & x=\frac{1}{2}, y=\frac{1}{5} \text { or } x=\frac{1}{3}, y=\frac{1}{4}, \\ 0.6, & x=\frac{1}{2}, y=\frac{1}{4} \text { or } x=\frac{1}{5}, y=\frac{1}{3}, \\ |x-y|, & x, y \in B \text { or } x \in A, y \in B .\end{cases}
$$

Then $(X, d)$ is a Branciari type generalized metric space, but it is not a metric space. In fact,

$$
0.6=d\left(\frac{1}{2}, \frac{1}{4}\right)>d\left(\frac{1}{2}, \frac{1}{3}\right)+d\left(\frac{1}{3}, \frac{1}{4}\right)=0.5
$$

Let $T: X \rightarrow X$ be defined by

$$
T x= \begin{cases}\frac{1}{5}, & x \in[1,2], \\ \frac{1}{4}, & x \in\left\{\frac{1}{2}, \frac{1}{3}, \frac{1}{4}\right\}, \\ \frac{1}{3}, & x=\frac{1}{5} .\end{cases}
$$

Define $\psi(t)=t, \varphi(t)=\frac{t}{5}, t \in[0,+\infty)$. Then $T$ satisfies

$$
\begin{aligned}
\psi(d(T x, T y)) \leq & \psi\left(a_{1} d(x, y)+a_{2} d(x, T x)+a_{3} d(y, T y)\right) \\
& -\varphi\left(a_{1} d(x, y)+a_{2} d(x, T x)+a_{3} d(y, T y)\right)
\end{aligned}
$$

for all $x, y \in X$, where $a_{1}=0.4, a_{2}=0.4, a_{3}=0.2$ and $T$ has a unique fixed point $x=\frac{1}{4}$. 


\section{Acknowledgements}

The authors are grateful to the anonymous referees for their good comments and valuable suggestions which helped to obtain the manuscript.

\section{Funding}

This work was supported by Science and Technology Research of Higher Education in Hebei province (ZD2019047).

\section{Availability of data and materials}

Not applicable.

\section{Competing interests}

The authors declare that they have no competing interests.

\section{Authors' contributions}

The authors contributed equally. Both authors read and approved the final version of the manuscript.

\section{Publisher's Note}

Springer Nature remains neutral with regard to jurisdictional claims in published maps and institutional affiliations.

Received: 7 October 2020 Accepted: 5 January 2021 Published online: 25 January 2021

\section{References}

1. Branciari, A.: A fixed point theorem of Banach-Caccippoli type on a class of generalized metric spaces. Publ. Math. Debrecen 57, 31-37 (2000)

2. Azam, A., Arshad, M.: Kannan fixed point theorem on generalized metric spaces. J. Nonlinear Sci. Appl. 1(1), 45-48 (2008)

3. Sarma, I.R., Rao, J.M., Rao, S.S.: Contractions over generalized metric spaces. J. Nonlinear Sci. Appl. 2(3), 180-182 (2009)

4. Lakzian, H., Samet, B.: Fixed point for $(\psi, \varphi)$-weakly contractive mappings in generalized metric spaces. Appl. Math. Lett. 25, 902-906 (2012)

5. Bari, C.D., Vetro, P.: Common fixed points in generalized metric spaces. Appl. Math. Comput. 218, 7322-7325 (2012)

6. Aydi, H., Karapinar, E.: Lakzian, H.: Fixed point results on a class of generalized metric spaces. Math. Sci. 6, 46 (2012)

7. Erhan, I.M., Karapinar, E., Sekulic, T.: Fixed points of $(\psi, \phi)$ contractions on rectangular metric spaces. Fixed Point Theory Appl. 2012138 (2012)

8. Liu, B., Chai, G.Q.: Fixed points theorem for weakly contractive mappings in generalized metric spaces. Hubei Shifan Xueyuan Xuebao 33(1), 60-65 (2013) (Chinese)

9. Cakic, N.: Coincidence and common fixed point theorems for $(\psi, \varphi)$ weakly contractive mappings in generalized metric spaces. Filomat 27(8), 1415-1423 (2013)

10. Proinov, P.D.: Fixed point theorems for generalized contractive mappings in metric spaces. J. Fixed Point Theory Appl. 22(21), 21-27 (2020)

11. Eshraghisamani, M., Vaezpour, S.M., Asadi, M.: New fixed point results on Branciari metric spaces. J. Math. Anal. 8(6), 132-141 (2017)

12. Eshraghisamani, M., Vaezpour, S.M., Asadi, M.: New fixed point results with $\alpha_{9 s p}$-admissible contractions on b-Branciari metric spaces. J. Inequal. Spec. Funct. 9(4), 101-112 (2018)

13. Younis, M., Singh, D., Asadi, M., Joshi, V.: Results on contractions of Reich type in graphical b-metric spaces with applications. Filomat 33(17), 5723-5735 (2019)

14. Karapinar, E.: Some fixed points results on Branciari metric spaces via implicit functions. Carpath. J. Math. 31(3), 339-348 (2015)

15. Ahmad, A.J., Karapinar, E.: Some common fixed point results in rectangular metric spaces. Int. J. Anal. 2013, Article ID 307234 (2013)

16. Karapinar, E., Pitea, A.: On $\alpha-\psi$-Geraghty contraction type mappings on quasi-Branciari metric spaces. J. Nonlinear Convex Anal. 17(7), 1291-1301 (2016)

17. Karapinar, E., Aydi, H., Samet, B.: Fixed points for generalized $(\alpha, \psi)$-contractions on generalized metric spaces. J. Inequal. Appl. 2014, 229 (2014)

18. Alghamdi, M.A., Chen, C.M., Karapinar, E.: A generalized weaker $(\alpha-\phi-\varphi)$-contractive mappings and related fixed point results in complete generalized metric spaces. Abstr. Appl. Anal. 2014, Article ID 985080 (2014)

19. Dutta, P.N., Choudhury, B.S.: A generalisation of contraction principle in metric spaces. Fixed Point Theory Appl. 2008 Article ID 406368, 8 pages (2008) 